\title{
Multiple myeloma in the very elderly patient: challenges and solutions
}

This article was published in the following Dove Press journal:

Clinical Interventions in Aging

15 April 2016

Number of times this article has been viewed

John Willan'

Toby A Eyre ${ }^{1,2}$

Faye Sharpley'

Caroline Watson'

Andrew J King ${ }^{1,3}$

Karthik Ramasamy ${ }^{1,4}$

'Department of Hematology, ${ }^{2}$ Early Phase Clinical Trial Unit, Oxford University Hospitals National Health Service (NHS) Foundation Trust, Churchill Hospital, Oxford, UK; ${ }^{3}$ Weatherall Institute of Molecular Medicine, John Radcliffe Hospital, Oxford, UK; ${ }^{4}$ National Institute for Health Research (NIHR) Biomedical Research Center Blood Theme, Oxford, UK
Correspondence: John Willan Department of Hematology, Oxford University Hospitals National Health Service (NHS) Foundation Trust, Churchill Hospital, Oxford OX3 7LE, UK Tel +44 I865 74l 84l

Email john.willan@ouh.nhs.uk

\begin{abstract}
Diagnosis and management of myeloma in the very elderly patient is challenging. Treatment options have vastly improved for elderly myeloma patients but still require the clinician to personalize therapy. In this paper, we offer evidence-based, pragmatic advice on how to overcome six of the main challenges likely to arise: 1) diagnosis of myeloma in this age group, 2) assessment of the need for treatment, and the fitness for combination chemotherapy, 3 ) provision of the best quality of supportive care, 4) choice of combination chemotherapy in those fit enough for it, 5) treatment of relapsed myeloma, and 6) provision of end of life care. With an increased burden of comorbidities and a reduced resilience to treatment and its associated toxicities, the management of myeloma in this age group requires a different approach to that in younger patients to maximize both quality and length of life.
\end{abstract}

Keywords: myeloma, elderly, diagnosis, treatment

\section{Introduction}

Plasma cell myeloma is a clonal disorder of malignant plasma cells and is a disease of the elderly, with a median age of onset of 70 years. ${ }^{1,2}$ Development of newer therapeutic agents over the last decade has led to improvements in survival in younger patients; $;, 4$ however, such benefits have yet to be realized in the very elderly ( $>80$ years) who continue to have poor outcomes. ${ }^{5}$ There are potential reasons for this. First, aging is associated with organ dysfunction, poorer resilience to physiological stressors, reduced functional status, and an increasing burden of comorbidities. ${ }^{6}$ Second, the elderly are at increased risk of frailty, a poorly defined syndrome characterized by a state of increased vulnerability to minor stressors with cumulative deficits in multiple physiological systems, resulting in an increased risk of hospitalization, dependency, and reduced life expectancy.

Elderly patients comprise a heterogeneous group of variable fitness from the very frail to the remarkably fit. ${ }^{7}$ Adequate assessment of fitness prior to treatment in this cohort is vitally important: inadequate assessment will inevitably lead to instances where frail patients are overtreated, and fitter patients are undertreated. In both situations, this can reduce the quality and length of life. The use of age and performance status (eg, the Eastern Cooperative Oncology Group [ECOG] score) alone is unsatisfactory, and there is a clear benefit to using geriatric assessment scores combining factors such as age, comorbidity burden, and assessments of functional status. ${ }^{8}$ Further increasing the difficulties in managing myeloma in this group is the paucity of clinical trial data. Stringent trial entry criteria typically exclude the majority of very elderly patients due to reduced performance status, comorbidities, or organ dysfunction. ${ }^{9}$ Trial candidates over the age of 80 , therefore, tend to be unusually fit and are not representative of the typical very elderly patient. 


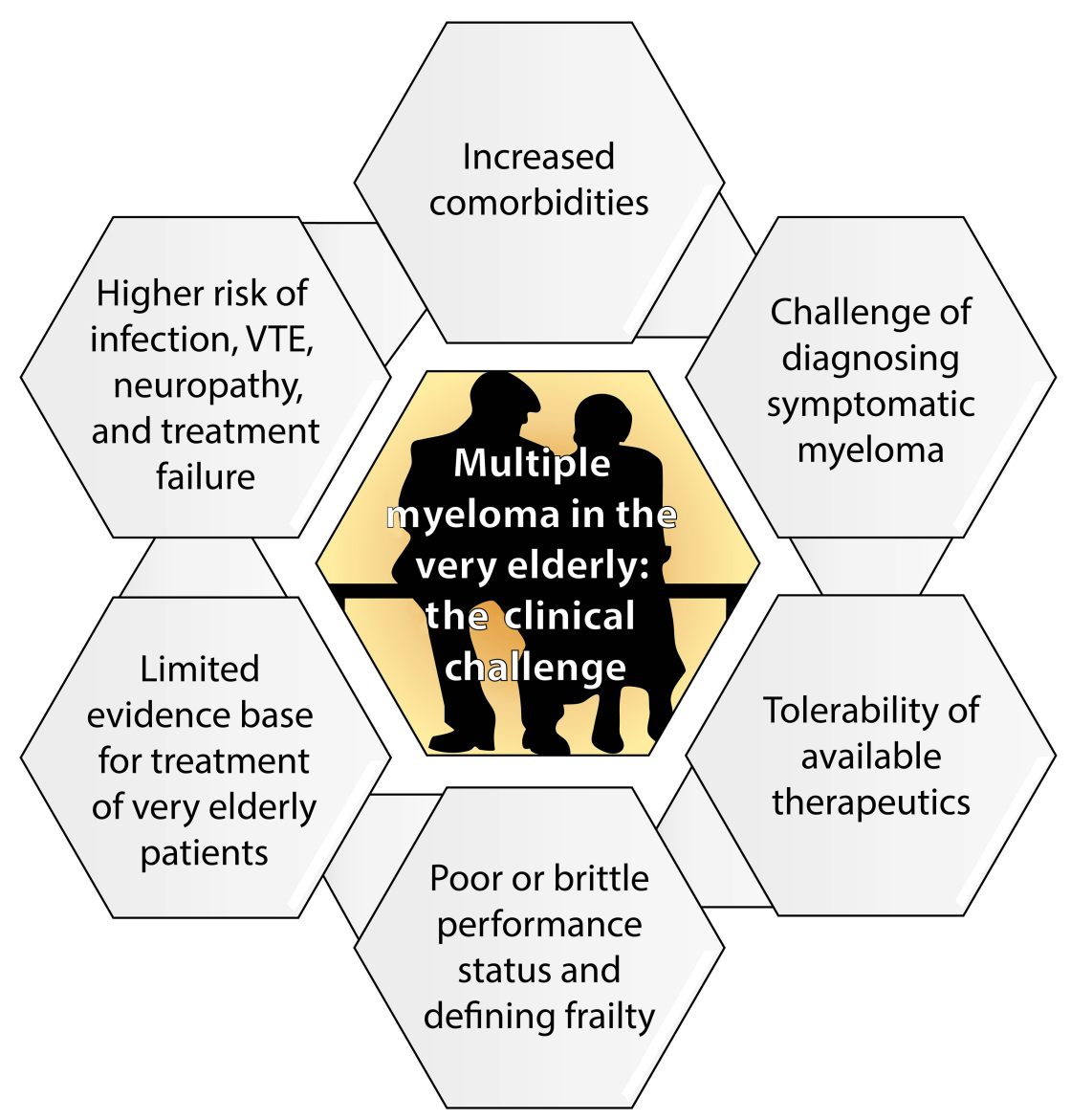

Figure I The challenges of diagnosis and treating the very elderly patient with multiple myeloma. Abbreviation: VTE, thromboembolism.

Reaching a diagnosis of myeloma, assessing the need for treatment, and choosing the relative treatment intensity in the very elderly are consequently highly complex (Figure 1). This review will address six main challenges to clinicians treating myeloma in the very elderly and discusses the strategies to overcome them.

\section{Challenge I - How is multiple myeloma diagnosed in the very elderly?}

Myeloma is preceded by an asymptomatic monoclonal gammopathy of undetermined significance (MGUS) in all patients, although only a small proportion of myeloma patients have this diagnosed, with the vast majority presenting de novo. In those diagnosed with MGUS, most do not progress to myeloma: a paraprotein is found in $4 \%-5 \%$ of people in their $80 \mathrm{~s},{ }^{10}$ whereas the incidence of myeloma in this group is only 40 per $100,000 .{ }^{11}$ It is therefore of fundamental importance to consider whether a patient has myeloma or incidental MGUS with unrelated organ dysfunction (Figure 2).
Myeloma can present with a plethora of clinical features including unexplained anemia, bone pain, hypercalcemia, renal dysfunction, fatigue, spinal cord compression (SCC), recurrent bacterial infections, and rarely, symptoms of hyperviscosity. ${ }^{12,13} \mathrm{Up}$ to $40 \%$ of myeloma patients present acutely with unexplained renal impairment, SCC, fracture, or profound hypercalcemia. A subset of patients present with a paraprotein or light chain excess and a full set of "CRAB" criteria (HyperCalcemia, Renal impairment, Anemia, and Bone lesions). In such patients, the diagnosis is usually considered early and rapidly confirmed, and so the treatment can begin promptly under the direction of a hematologist.

Diagnosis of a very elderly patient presenting insidiously with mild anemia, renal impairment, or bone pain, all of which can often be attributed to another etiology, requires detailed evaluation. There is often a lengthy delay between symptom onset and diagnosis of myeloma, with the average duration being around 6 months. ${ }^{14}$ Access to secondary care for assessment is significantly delayed, with over $50 \%$ of newly diagnosed patients requiring three visits to a general practitioner (GP) before a referral is made. ${ }^{15}$ 

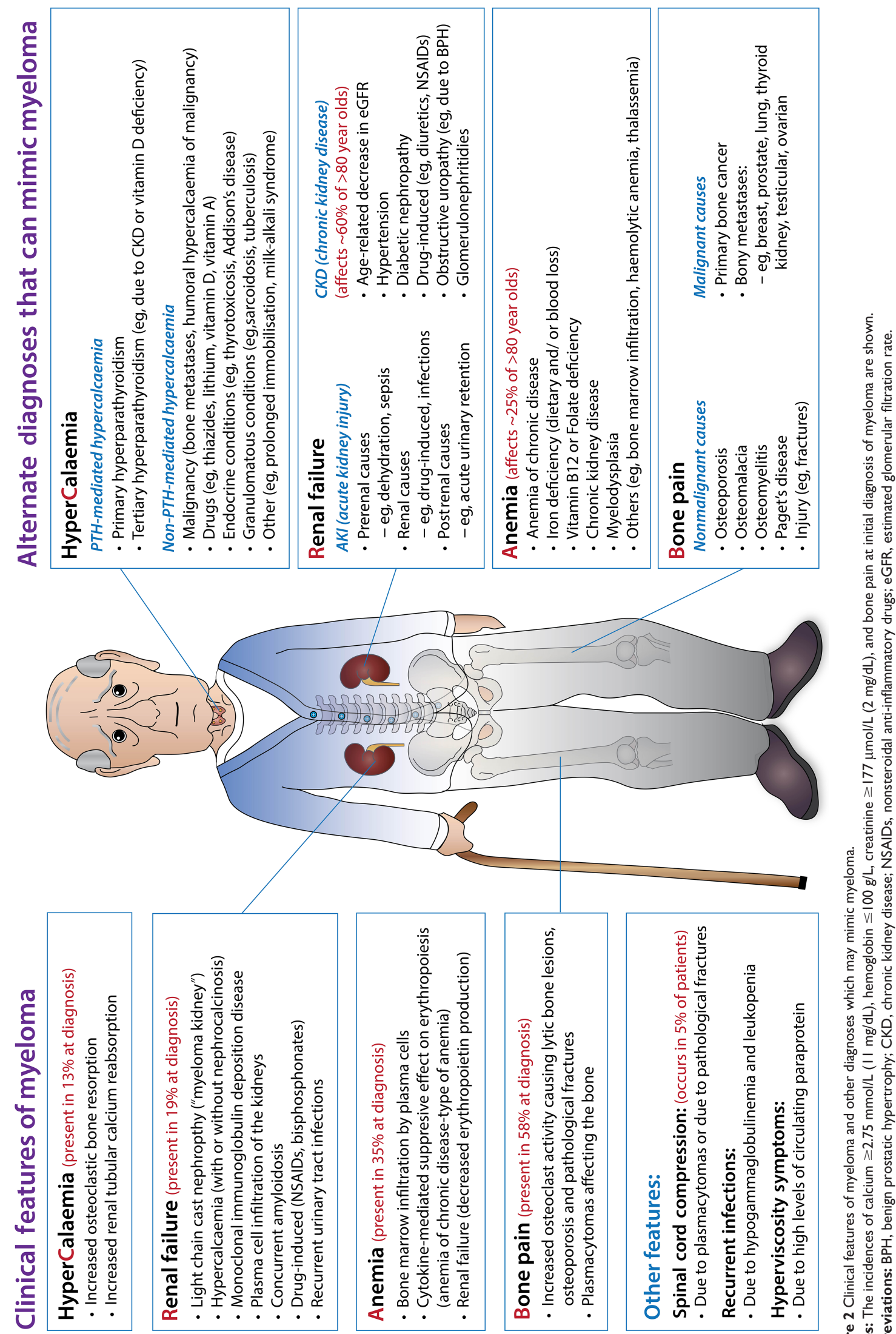

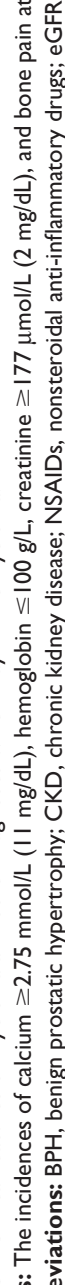


Table I Investigations required in very elderly patients with possible plasma cell myeloma

\begin{tabular}{|c|c|c|}
\hline \multicolumn{3}{|l|}{ To make the diagnosis } \\
\hline First-line investigations & $\begin{array}{l}\mathrm{FBC}, \mathrm{U \& E} \text {, creatinine, calcium, protein } \\
\text { electrophoresis, urinary electrophoresis, SFLC ratio }\end{array}$ & $\begin{array}{l}\text { This should be performed in all patients suspected of } \\
\text { having myeloma }\end{array}$ \\
\hline To exclude mimics & PTH, blood film, hematinics, fasting glucose & $\begin{array}{l}\text { As appropriate to exclude other causes for symptoms and } \\
\text { abnormal blood results }\end{array}$ \\
\hline Radiology & $\begin{array}{l}\text { Skeletal survey } \\
\text { MRI spine and pelvis in patients with back pain }\end{array}$ & $\begin{array}{l}\text { All patients, but in the very frail may just target areas of } \\
\text { pain }\end{array}$ \\
\hline \multicolumn{3}{|l|}{ To confirm diagnosis } \\
\hline Bone marrow & Aspiration and trephine & $\begin{array}{l}\text { All patients, but consider omitting trephine in frail patients, } \\
\text { particularly if combination chemotherapy is inappropriate }\end{array}$ \\
\hline Bone marrow & Flow cytometry & Assessment of clonality \\
\hline \multicolumn{3}{|c|}{ To assess disease status } \\
\hline Blood & $\beta_{2}$-microglobulin, albumin, LDH & To assess tumor burden and prognosis \\
\hline Bone marrow & FISH panel & To assess prognosis \\
\hline
\end{tabular}

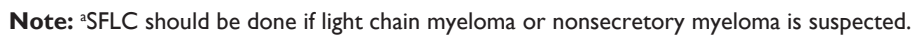

Abbreviations: FBC, full blood count; U\&E, urea and electrolytes; SFLC, serum free light chains; PTH, parathyroid hormone; LDH, lactate dehydrogenase; FISH, fluorescence in situ hybridization; MRI, magnetic resonance imaging.

Normally, GPs or general medical teams discover an incidental paraproteinaemia or note an abnormal imaging result; hematologists are then involved for advice regarding interpretation and further investigation. A thorough evaluation in secondary care is often required in this situation. In such situations, further investigations listed in Table 1 should be considered.

Eighty percent of patients with myeloma have a detectable paraprotein; the vast majority of the remainder have light chain only myeloma (where the malignant clone produces a monoclonal light chain rather than the full immunoglobulin molecule). ${ }^{14}$ Less than $2 \%$ of patients have nonsecretory myeloma where there is neither a paraprotein nor significant light chain production. Immunoparesis, where the normal immunoglobulin levels are decreased (due to impaired production of normal functioning plasma cells), leads to an increased risk of infection in myeloma patients and can provide an important clue to light chain or nonsecretory myeloma when a paraprotein is not present.

Diagnosing myeloma (Table 2) requires the demonstration of a clonal population of plasma cells either within the bone marrow, or less commonly, within a bony or extramedullary plasmacytoma. Bone marrow sampling is generally well tolerated in the elderly; however, its necessity must be carefully considered in the frail patient. Such patients may be at increased risk of side effects including significant bleeding, not only as they may be unable to lie in the optimal position and are more likely to be taking antiplatelet agents, but also because a paraprotein can interfere with fibrin production. The relative risk of bleeding must, therefore, be carefully considered. Osteoporosis in a proportion of myeloma patients could make trephine biopsy sampling difficult. In a frail elderly patient, the clinician may choose to perform aspiration alone, or alternatively to omit the test altogether when palliation is considered.

Patients are routinely staged according to the International Staging System for myeloma using a combination of albumin and $\beta_{2}$-microglobulin to assess overall prognosis. ${ }^{16}$ Certain cytogenetic abnormalities are associated with poor prognosis including deletions of chromosome 17p (TP53 deletion), $t(4 ; 14)$ and $t(14 ; 16)$ and these are normally assessed by fluorescence in situ hybridization (FISH) at diagnosis. ${ }^{17}$ A retrospective analysis of outcomes in older patients with chromosomal abnormalities (median age 72 years; range 66-94 years) demonstrates that these high risk cytogenetic features predict poor outcomes regardless of age. ${ }^{18}$

There should be a low threshold for magnetic resonance imaging (MRI) of the whole spine in proven cases of myeloma

Table 2 Diagnosis of symptomatic myeloma

Clonal population of plasma cells $>10 \%$, or biopsy proven plasmacytoma plus one or more of

Evidence of end-organ damage that can be attributed to the plasma cell disorder

Hypercalcemia: calcium $>0.25 \mathrm{mmol} / \mathrm{L}$ above normal range

or $>2.75 \mathrm{mmol} / \mathrm{L}$

Renal impairment: creatinine clearance $<40 \mathrm{~mL} / \mathrm{min}$ or creatinine

$>177 \mu \mathrm{mol} / \mathrm{L}$

Anemia: hemoglobin $>20 \mathrm{~g} / \mathrm{L}$ below lower limit of normal or $<100 \mathrm{~g} / \mathrm{L}$

Bony lesions: one or more osteolytic lesions on plain XR/CT/PET-CT

Myeloma defining events in the absence of end-organ damage

Clonal plasma cell population $>60 \%$

Ratio of involved: uninvolved SFLC $>100$ with involved SFLC $>100 \mathrm{mg} / \mathrm{L}$

$>$ I focal lesion of $>5 \mathrm{~mm}$ in size on MRI

Note: Data from Rajkumar et al. ${ }^{62}$

Abbreviations: PET-CT, positron emission tomography-computed tomography; SFLC, serum free light chains; MRI, magnetic resonance imaging. 
with back pain to help guide areas suitable for radiotherapy or vertebroplasty, assess for SCC, and exclude multifocal plasmacytomas. Whole-body imaging including MRI and positron emission tomography-computed tomography (PET-CT) may be of value, alongside close monitoring in clinic to determine the trajectory and pace of organ dysfunction. A PET-CT requires a patient to move to and from a bed unaided; an important consideration in a very elderly patient.

\section{Challenge 2 - How should the need for treatment and the fitness for treatment be assessed in the very elderly?}

MGUS patients are normally monitored in primary care with pre-agreed guidelines for re-referral and specialist hematology input as required. ${ }^{17}$ Similarly, patients with smoldering myeloma (patients meeting the diagnostic criteria for myeloma in terms of their paraprotein level and/or marrow plasma cell percentage but without resulting end-organ damage) do not require treatment, although they have a $50 \%$ risk of requiring treatment over the next 5 years. ${ }^{19,20}$ Patients with high-risk smoldering myeloma should be closely monitored or recruited into appropriate clinical trials unless the patient is very frail and active treatment would be inappropriate.

The decision to initiate combination chemotherapy should be taken by a specialist myeloma multidisciplinary team considering all available diagnostic and clinical information, and in discussion with the patient and their family. Once endorgan damage attributable to myeloma has been identified, treatment is recommended.

Comprehensive geriatric assessment (CGA) tools have been created to help guide treatment decisions in elderly patients with cancer. ${ }^{8}$ A specific CGA has been developed for myeloma which aims to divide patients at diagnosis into groups of variable fitness to help guide treatment schedules. ${ }^{21}$ This score was developed by pooled analysis of 869 patients prospectively treated in three different clinical trials in which several methods of assessment had been performed at baseline. ${ }^{22-24}$ The most useful tools to predict outcome were the Katz Activities of Daily Living, the Lawton Instrumental Activities of Daily Living, and the Charlson Comorbidity Index. These were combined with patient age to categorize individuals into three groups: fit, intermediate fitness, and frail. The single factor that best predicted a reduced overall survival (OS) was age over 80 years (hazard ratio [HR]: 2.4). This particular tool automatically allocates the maximum score for frailty to all patients over 80 years based solely on their age and therefore is unable to distinguish the fitter from the more frail. ${ }^{21}$ An alternative CGA tool combining performance status with assessment of renal and respiratory function and validated in patients with myeloma may be a useful alternative means of assessment. ${ }^{25}$ Other geriatric assessment tools have been developed and applied to hematological cancers in general, and it is likely that a geriatric assessment score will be incorporated into future international trials. ${ }^{26-28}$ There is a clear need to gather further prospective evidence in this older age group, and to develop and validate new scoring systems that may guide practice in the future.

Pragmatically, the treating clinician should take into account age, performance status, a CGA tool, and importantly the views of the patient and their family when planning treatment. These should be combined with their own clinical judgment to arrive at an overall assessment of which treatments should be offered. Regardless of their fitness for combination chemotherapy, all elderly patients require careful management of both disease-related and treatment-related symptoms. A principal aim in the very elderly is the maintenance or improvement of quality of life with improved OS.

Combination chemotherapy may be more effective in controlling symptoms such as lytic bone pain than traditional palliative care, and such regimens can be offered with this intent. If chemotherapy regimens are carefully attenuated, then it is possible to reduce drug discontinuations, which commonly occur in the very elderly patients population. ${ }^{21}$ Despite this, there will be a small portion of the very elderly who are not fit even for the most attenuated treatment. Such patients should be identified early and managed according to the best principles of palliative care, treating symptoms while minimizing invasive investigations.

\section{Challenge 3 - How can supportive care best be provided in the very elderly?}

Providing appropriate supportive care to myeloma patients is of vital importance, both for symptom control and management of disease-specific complications. Input from specialists in pain medicine, orthopedic surgery, interventional radiology, and palliative care is often required.

\section{Management of skeletal complications and pain}

Myeloma-related skeletal complications in the elderly can be severe and debilitating; a variety of treatment approaches may be combined in addition to disease modification. Importantly, SCC occurs in $5 \%$ of patients with myeloma and may be the 
presenting problem. High-dose dexamethasone should be commenced upon suspicion and definitive treatment planned with either surgery or radiotherapy after appropriate imaging; radiotherapy is pragmatically favored in the very elderly.

Provision of adequate analgesia is vital and specialist pain team input may be required. Paracetamol is safe, but nonsteroidal anti-inflammatory drugs (NSAIDs) should be used with extreme caution due to the risk of nephrotoxicity. Opiates are often needed, but their side effects are more pronounced in the elderly and as such doses should be titrated carefully.

Radiotherapy and occasionally orthopedic surgery may be appropriate in the management of skeletal disease. Low-dose radiotherapy may be effective in the treatment of isolated painful bony lesions; typically, only a single dose ( $8 \mathrm{~Gy}$ ) is required for adequate control. ${ }^{29}$ Kyphoplasty or vertebroplasty may help when vertebral compression fractures results in pain unresponsive to analgesia or to stabilize vertebrae at risk of fracture.

Bisphosphonates reduce the risk of new skeletal-related events and are routinely given to all patients with symptomatic myeloma. Data from the Myeloma IX trial suggested an improved OS of zolendronic acid over clodronate, ${ }^{30}$ and this is generally preferred to pamidronate, which takes longer to infuse. A pragmatic approach of infrequent infusions is sometimes needed in treating elderly myeloma patients who may find monthly visits to hospital for infusions tiring and impractical. Intravenous (IV) bisphosphonates are contraindicated in chronic renal impairment where the creatinine clearance is $<30 \mathrm{~mL} / \mathrm{min}$.

Osteonecrosis of the jaw is a rare complication of bisphosphonate treatment; the risk is higher in patients with poor dentition, following invasive dentistry, and with IV preparations. Patients should be reviewed by a dentist pretreatment, and dental work should be avoided where possible once treatment is initiated. In patients who achieve complete remission with treatment, bisphosphonates can reasonably be stopped after 2 years, although this is unfortunately rare in the elderly. Calcium and vitamin D supplementation should be routinely given to all patients taking bisphosphonates to avoid hypocalcaemia, but care should be taken that their use does not exacerbate hypercalcemia in certain patients.

\section{Anemia}

Anemia is one of the hallmark features of myeloma, present in $35 \%$ at diagnosis. It may be exacerbated by chemotherapy. Management includes judicious red blood cell transfusion, consideration of IV iron infusion, and in selected patients, erythropoietin-stimulating agents. ${ }^{12}$

\section{Renal failure}

Renal failure in myeloma is multifactorial in nature, occurring due to damage to renal tubules by free light chains, inappropriate NSAID usage, dehydration, hypercalcemia, and infection. Renal function declines with age, and so the elderly are less resilient to such insults. Dexamethasone should be commenced as soon as multiple myeloma is suspected; prompt treatment can reverse renal dysfunction in about $50 \% .{ }^{31}$ If dialysis is indicated, a frank discussion involving the patient, their family, renal physicians, and myeloma specialists is necessary to determine the appropriateness of such an intervention.

\section{Thromboembolic disease}

Patients with myeloma are at increased risk of venous thromboembolism (VTE). ${ }^{32}$ VTE is more common with increasing age, and the use of the immunomodulatory agents (IMiDs) thalidomide and lenalidomide further increase this risk. ${ }^{33}$ Low-molecular-weight-heparin prophylaxis should be considered in patients judged to be at high risk; however, this can be logistically difficult in the very elderly due to poor eyesight, lack of dexterity, and low confidence with self-injecting. Aspirin may be a suitable, evidence-based compromise. ${ }^{34}$ Novel oral anticoagulants are used in clinical practice with limited evidence as prophylactic treatment for patients on IMiDs, due to their convenience.

\section{Infectious complications}

Analysis of registry data from 1980 to 2002 concluded that $10 \%$ of myeloma patients die of infection within 60 days of diagnosis due to deficits in cellular and humoral immunity. ${ }^{35}$ Elderly patients are particularly prone to infection. Prophylactic fluconazole (to prevent candidiasis) and aciclovir (to prevent herpes simplex and/or zoster) are typically coprescribed with chemotherapy. There is no good evidence for the use of antibacterial prophylaxis at present. The UKwide "Tackling early morbidity and mortality in myeloma" (TEAMM) trial is currently recruiting, and will determine whether primary fluoroquinolone prophylaxis is beneficial. Clarithromycin has some anti-myeloma properties and has been used in some experimental combination regimes, while providing antibacterial prophylaxis. ${ }^{36}$ Neutropenia may be managed by the use of granulocyte colony stimulating factor. 


\section{Challenge 4 - How should fitter very elderly patients be treated with combination chemotherapy?}

\section{Summary of trial evidence}

Large, multicenter, randomized controlled trials published in high-impact journals lay the foundation for an evidence-based approach to treating patients who are not deemed eligible for an autologous stem cell transplant (ASCT) in first remission (Table 3). ${ }^{37-41}$ This is typically defined as those over 65 years or those younger than 65 years with prohibitive comorbidities. These large trials are typically performed at major tertiary referral centers across Europe and America, and as such it is unsurprising that the median age in such trials is approximately 70 years, with only between a third to a quarter of patients over 75 years. As a result, extrapolating the evidence base to the very elderly must be done with caution. The data do, however, allow some conclusions to be drawn to guide clinicians in the management of very elderly patients.

Novel agents are now commonly used in all age groups in myeloma. Although steroid and alkylator therapy formed the backbone of induction treatment for many years, the IMiDs thalidomide and lenalidomide and the first-generation proteasome inhibitor bortezomib (velcade) have significantly changed the treatment landscape and outcomes over the last decade.

A large meta-analysis of six clinical trials has shown a clear survival benefit from the use of thalidomide in addition to MP (melphalan, prednisolone) in those unfit for ASCT. ${ }^{42}$ Thalidomide, however, is known to be poorly tolerated at high doses, particularly in the very elderly. Constipation, cardiac events, excessive somnolence, peripheral neuropathy (PN), and VTE are well-described side effects that are prevalent and poorly tolerated in the very elderly. ${ }^{42}$ A substantial proportion of patients across these six trials either had thalidomide stopped prematurely or its dose reduced. As such, it is critical to assess tolerability and use appropriate dosage (typically $50-100 \mathrm{mg}$ once daily maximum) in the very elderly.

The randomized controlled VISTA trial investigated whether the addition of bortezomib (velcade) to MP (VMP) improved outcomes in those unfit for ASCT as first-line treatment. ${ }^{38}$ The addition of bortezomib showed a significantly improved duration of remission, progression-free survival (PFS), and ultimately 5-year OS (56.4 months vs 43.1 months). ${ }^{43}$ Notably, the VMP schedule was protracted, requiring regular visits to hospital for IV bortezomib for up to 54 weeks. The length of any regimen and outpatient time investment given must be considered in a group with a shorter all-cause life expectancy. In view of this, many clinicians now use subcutaneous bortezomib due to evidence of reduced PN, increased speed of delivery, and equivalent efficacy. ${ }^{44}$

The UPFRONT trial addresses whether triple therapy is of benefit when bortezomib is used in the very elderly ${ }^{45}$ The trial included a higher proportion of patients with comorbidities and elderly patients ( $42 \% \geq 75$ years and $18 \% \geq 80$ years) and recruited from US community-based settings as opposed to large, tertiary referral units. Velcade-dexamethasone (VD) was compared with VTD and VMP. All patients who responded to induction received bortezomib maintenance. This is the largest study to date that intentionally reflects the elderly population in the "real-world" clinic setting. There were no differences between the three arms in terms of median PFS or median OS (OS: VD 49.8 months vs VTD 51.5 months vs VMP 53.1 months; global $P=0.46$ and $P=0.79$ ). The most toxicities were seen in the VTD arm. Velcade maintenance resulted in limited additional toxicity compared to induction, while sustaining responses in $89 \%$.

Lenalidomide is a more potent IMiD than thalidomide, and recent trials have investigated its use upfront in those unfit for ASCT. In the largest and most relevant trial, melphalan, prednisolone, thalidomide (MPT; $\mathrm{n}=547$ ) was compared with up to 18 cycles (28 day) of lenalidomide-dexamethasone (LD18) $(\mathrm{n}=541)$ and continuous lenalidomide-dexamethasone (cLD) $(\mathrm{n}=535)$ to progression. cLD proved superior in terms of PFS compared to both MTP and LD18 (median PFS 25.5 months with cLD vs 20.7 months with LD18 vs 21.2 months with MPT; $P<0.001$ with cLD compared to both MTP and LD18). The improved PFS with cLD resulted in a superior OS compared to MTP (4-year OS 59\% vs 51\%; HR, 0.78; $P=0.02$ ) but not compared to LD18 (4-year OS 59\% vs 56\%; HR, $0.90 ; P=0.31$ ). This trial supports the use of continuous lenalidomide therapy. Low-dose dexamethasone with lenalidomide is associated with reduced toxicity and improved survival compared to high-dose dexamethasone. ${ }^{46}$ Where LD is used continuously in the very elderly, it is critical to monitor for adverse effects, particularly from long-term steroid exposure. No increase in second primary malignancy was noted in this trial in the lenalidomide arms. Recently presented updated data from the FIRST trial show that RD continuous therapy performs poorly in patients with high-risk disease, compared to those with standard risk. ${ }^{47}$ SWOG trial S0777 trial supports this finding, as patients randomized to VRD had a better OS in comparison to those treated with $\mathrm{cLD}{ }^{48}$ 


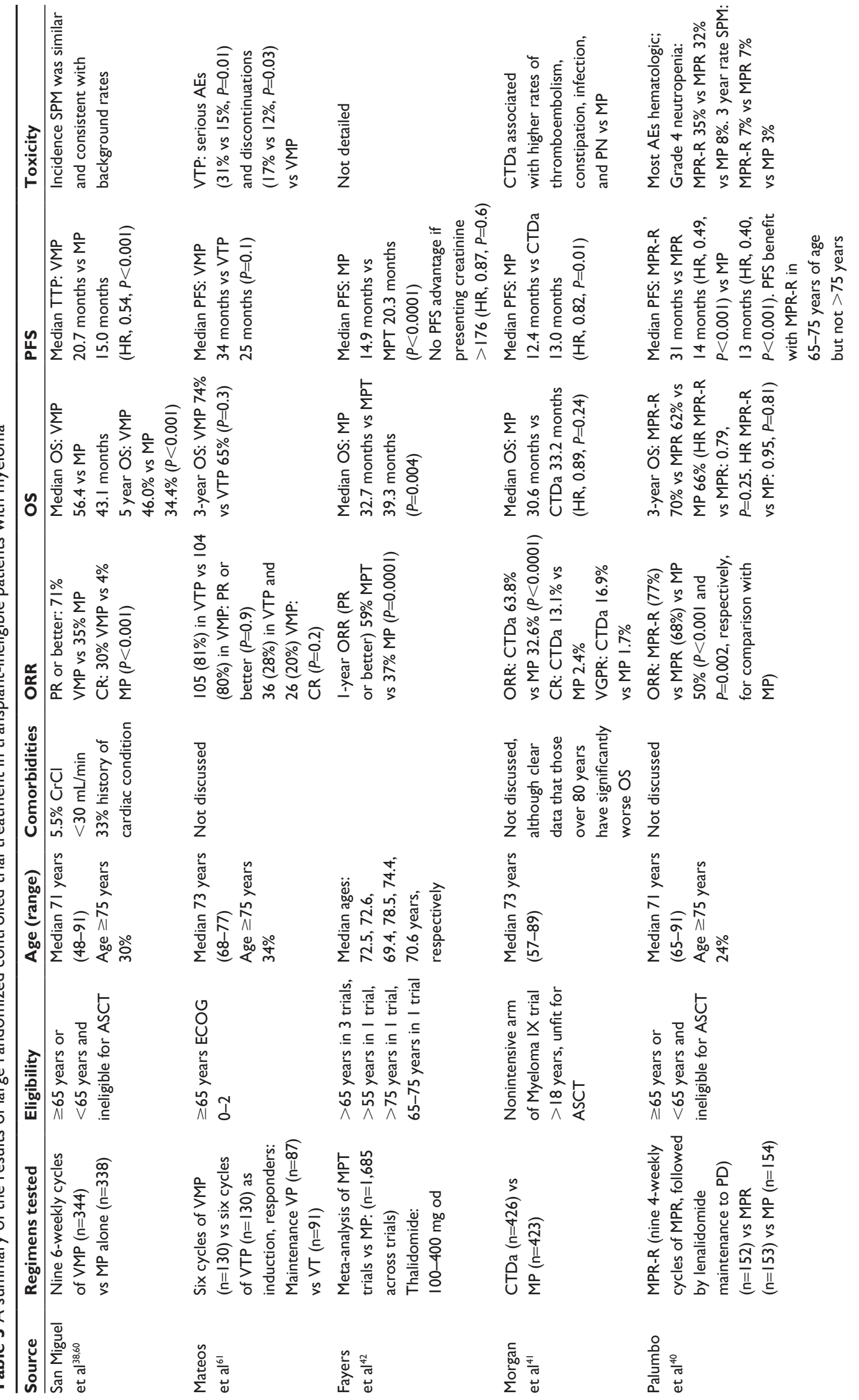




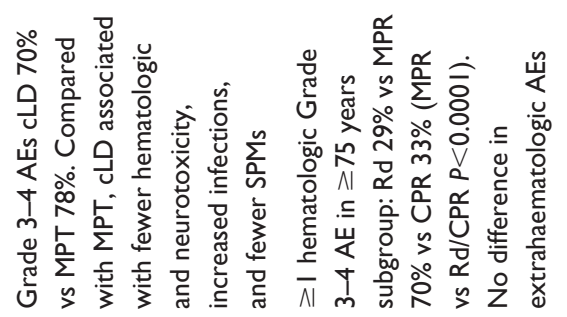

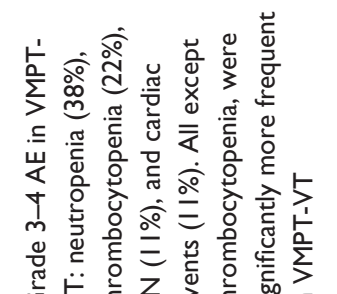

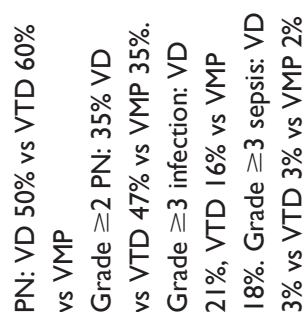

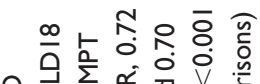

$\sum$ U⿺辶一

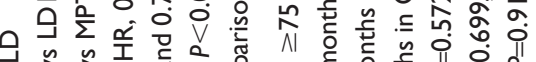

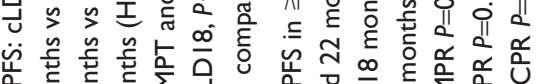

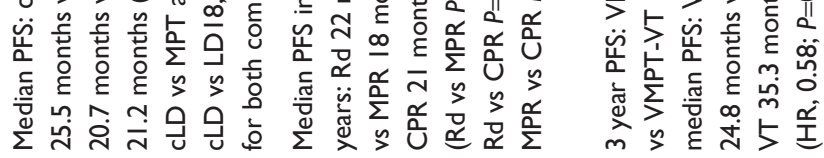

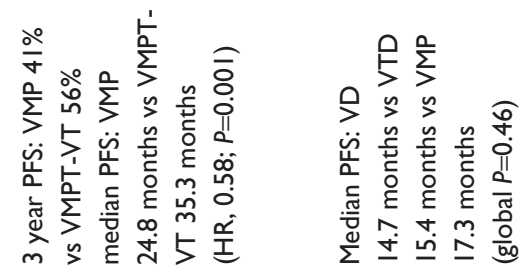

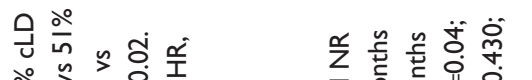

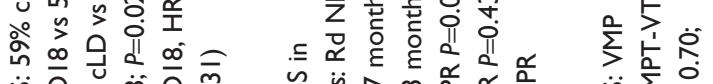

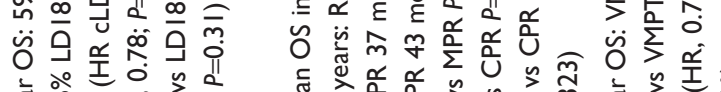

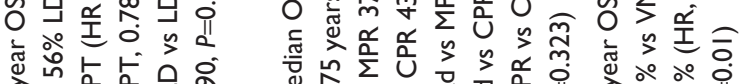

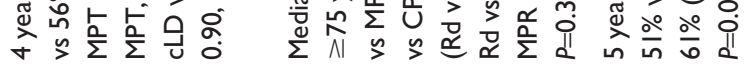

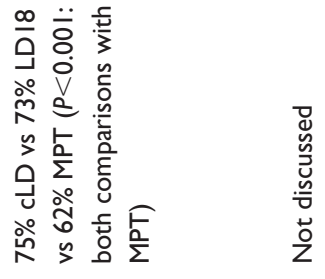

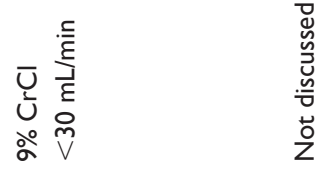

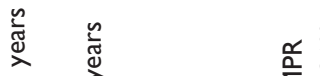



这栾总 总

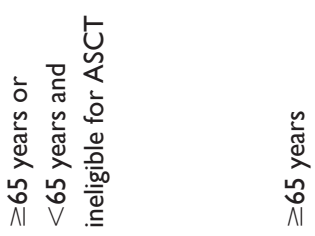

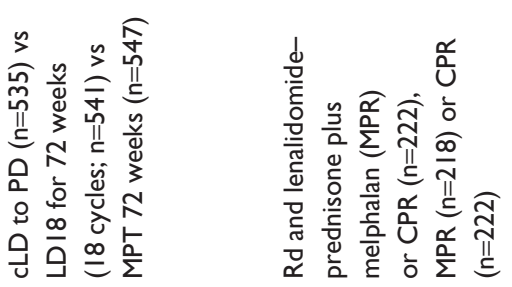

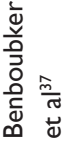

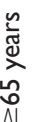

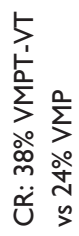

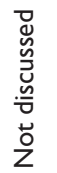
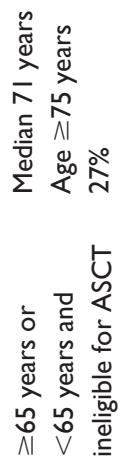

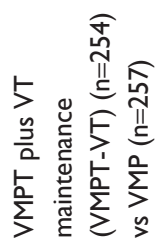

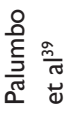

$\stackrel{\rho}{5} \sum_{>}^{0}$

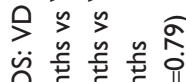

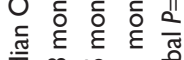

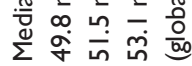

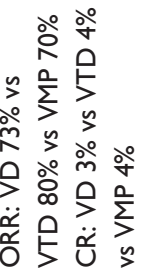

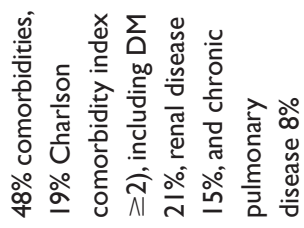

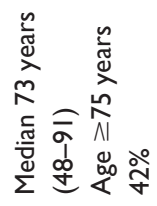

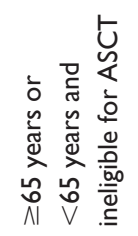

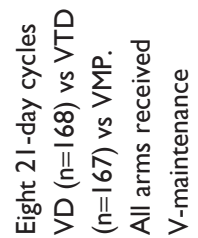

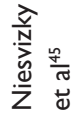

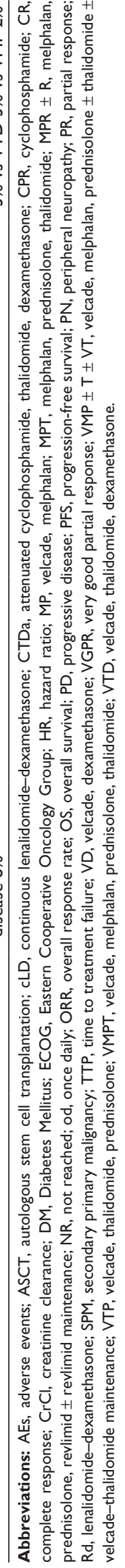


Table 4 Choice of therapeutic agent in patients with comorbidity

\begin{tabular}{ll}
\hline Comorbid condition & Advice on therapeutic agent \\
\hline Renal impairment & $\begin{array}{l}\text { Prefer bortezomib-based regimes } \\
\text { Need to reduce dose if use lenalidomide }\end{array}$ \\
Polyneuropathy & Avoid or reduce dose if use bortezomib \\
Cardiac arrhythmia/ & Caution with thalidomide and high-dose \\
dysfunction & steroids \\
Diabetes & Caution with high-dose steroids \\
Psychiatric/behavioral & Caution with high-dose steroids \\
problems & \\
Bone marrow & Caution with cytoreductive drugs \\
insufficiency & Consider single-agent dexamethasone \\
Poor immune function & Caution with cytoreductive drugs \\
Poor cognitive function & Consider subcutaneous and/or \\
or compliance & hospital-delivered regimes \\
\hline
\end{tabular}

A subgroup analysis of patients over 75 years in a large trial comparing lenalidomide-dexamethasone (low dose; Rd) and lenalidomide-prednisone plus melphalan (MPR) or cyclophosphamide (CPR) was recently published in abstract form. ${ }^{49}$ The addition of an alkylating agent provided no additional benefit beyond lenalidomide-dexamethasone alone, and $\mathrm{Rd}$ appears to have a survival advantage compared to MPR (median OS not reached in Rd vs 37 and 43 months in the MPR and CPR arms, respectively [Rd vs MPR $P=0.04$; $R d$ vs CPR $P=0.430]$ ). The OS difference was primarily due to a higher efficacy of salvage treatment.

\section{Recommendations for first-line treatment}

On the basis of age, CGA, performance status, and overall clinical assessment, it is possible to stratify patients into fit patients suitable for two or three drug combination therapies, frail patients requiring significantly attenuated therapies, and those suitable only for palliative care. In general, given the greater toxicity with thalidomide and alkylating agents, these are avoided in this age group whenever possible. Preexisting comorbidities as well as disease characteristics must be considered when selecting a treatment regime (Table 4).

This trial data support the use of lenalidomidedexamethasone as first-line treatment for fit patients continued until disease progression. This has the advantage of limiting visits to hospital to outpatient visits, and it can be used in pre-existing PN. In patients with significant renal disease, or aggressive disease requiring rapid paraprotein reduction, subcutaneous bortezomib-dexamethasone is preferred.

In frail patients, lenalidomide may also be used as initial therapy, although dose reduction to typically no more than
$15 \mathrm{mg}$ once daily is necessary. The dexamethasone dose should also be reduced, to around $10 \mathrm{mg}$ once per week. Careful monitoring is necessary, and granulocyte colony stimulating factor can be used to minimize neutropenia. If bortezomib-dexamethasone is selected, dexamethasone should be dose reduced, and patients should be carefully monitored for bortezomib toxicity.

In patients with severe cognitive impairment or very poor functional status, palliation may be preferred. In such situations, low-dose steroids may offer symptom relief.

\section{Challenge 5 - How should relapse be treated in the very elderly?}

Patients with asymptomatic serological relapse can have treatment delayed until they develop evidence of organ dysfunction, akin to the approach described with asymptomatic myeloma. A rapidly rising paraprotein (doubling in 2 months) is an indication of progressive disease requiring re-treatment, and as such, serological relapse should be monitored closely. The goals of treatment are again to improve quality of life and survival. Further treatment may be inappropriate depending upon the patient's frailty and wishes.

Novel agents again form the mainstay of treatment at relapse. Rechallenge with lenalidomide or bortezomib may be appropriate if a response $>12$ months was previously achieved. ${ }^{50}$ In refractory disease or short-term response only ( $<12$ months), switching regimes (lenalidomide-based with bortezomib-based) is recommended. Good evidence exists for lenalidomide in relapsed disease, ${ }^{50}$ with a smaller study indicating benefit in patients over 75 years with a median PFS of 14 months. ${ }^{51}$ Data also support the use of bortezomib at relapse, ${ }^{52,53}$ with one small trial indicating a clear benefit in elderly patients. ${ }^{54}$

Patients relapsing after bortezomib and lenalidomide may occasionally still be fit enough for further treatment. Treatment in clinical trials should be considered in such situations.

There are a number of emerging treatments that are likely to benefit elderly patients in the future. Carfilzomib, a novel proteasome inhibitor, is effective in newly diagnosed and relapsed myeloma. ${ }^{24,55}$ It is associated with a reduced toxicity compared to bortezomib (particularly PN) and may therefore take the place of bortezomib first line in the future, although its use should be avoided in patients with significant pre-existing cardiac disease. Ixazomib, another new proteasome inhibitor, has the advantage of oral administration. A Phase III trial has recently reported data in abstract form demonstrating improved survival in relapsed/refractory patients when ixazomib is used together with $\mathrm{Rd}$ in 
comparison to Rd alone. The side-effect profile was overall felt to be acceptable, although PN rates were increased in the ixazomib arm. ${ }^{56}$ The monoclonal antibodies daratumumab (an anti-CD38 monoclonal antibody) and elotuzumab (an anti-CS1 monoclonal antibody) are also in advanced clinical trials. ${ }^{57,58}$ Both have good tolerability and are also likely to particularly benefit the elderly should they prove effective in Phase III clinical trials. Relapsing disease often impacts on performance status of patients; in selected individuals, palliation may be appropriate.

\section{Challenge 6 - the management of end-of-life care}

"End of life" is now considered as the last 12 months of life $\mathrm{e}^{59}$ (National Institute for Health and Care Excellence), and for some elderly myeloma patients, this may be from the point of diagnosis. A holistic needs assessment is therefore a vital tool from the outset, allowing patients to openly convey their needs and wishes. An honest discussion about prognosis both at diagnosis and each relapse is crucial for the patient and their family to make the appropriate choices with regard to their treatment. It is becoming increasingly difficult to determine when a patient has reached the terminal stages of their disease due to the increasing palate of treatment options and trials available, and patients are sometimes treated until their last few days. Myeloma remains incurable, and so stopping chemotherapy and focusing on palliative care can be the most appropriate decision for the patient and their family. Advice from the palliative care team is often beneficial in the final stages as patients become increasingly weak and bedbound. Analgesia can be given subcutaneously, often in the form of syringe drivers to help control refractory pain and prevent periods of inadequate analgesia. Close communication between clinicians, nurse specialists, the palliative care team, social services, and GPs is of paramount importance to support the needs of the patient and their family.

\section{Conclusion}

Diagnosing and treating myeloma in the very elderly is challenging, requiring careful consideration of when to treat and how aggressively. A careful assessment of fitness for therapy must be conducted to allow treatment to be provided at an appropriate intensity. Close attention from a multidisciplinary team to provide adequate supportive care is essential to allow the patient to benefit from combination chemotherapies. Particular care must be taken to minimize toxicities, reducing doses if required to allow continuation of treatment when appropriate. As the population ages, the incidence of myeloma in the very elderly will increase. Novel and emerging therapies are likely to provide significant benefits to this patient group, with potent anti-myeloma activity combined with easier administration and lower toxicity. There is an urgent need to increase recruitment of patients in this age group to clinical trials to increase the evidence base and to allow the clinician and patient to make informed, evidence-based decisions on treatment strategies. Treating myeloma in the very elderly is challenging, but with judicious use of supportive and active treatments, compassionate and effective care can be provided to these patients.

\section{Acknowledgments}

TE is funded by the Julian Starmer-Smith research fund and has received honorarium from Takeda. AK is funded by Wellcome Trust. KR is supported by the NIHR Biomedical Research Centre (Oxford). The views expressed are those of the authors and not necessarily those of the Department of Health.

\section{Author contributions}

TE, JW, AK, CW, and FB wrote the manuscript. TE produced Table 3 and Figure 1. CW produced Figure 2. KR, TE, JW, and AK edited the manuscript. All authors contributed toward data analysis, drafting and revising the paper and agree to be accountable for all aspects of the work. All authors critically reviewed the manuscript and agreed on the final version.

\section{Disclosure}

TE has received consultancy honoraria from Janssen. KR has received honoraria from Jansenn, Celgene, Takeda and Amgen. The authors report no other conflicts of interest in this work.

\section{References}

1. Altekruse SF, Kosary CL, Krapcho M, et al. SEER Cancer Statistics Review, 1975-2007. Bethesda, MD: National Cancer Institute; 2010 Available from: http://seer.cancer.gov/csr/1975_2007/index.htm1. Accessed February 4, 2016.

2. Palumbo A, Bringhen S, Ludwig H, et al. Personalized therapy in multiple myeloma according to patient age and vulnerability: a report of the European Myeloma Network (EMN). Blood. 2011;118(17) 4519-4529.

3. Kumar SK, Rajkumar SV, Dispenzieri A, et al. Improved survival in multiple myeloma and the impact of novel therapies. Blood. 2008;111(5):2516-2520.

4. Kumar SK, Dispenzieri A, Lacy MQ, et al. Continued improvement in survival in multiple myeloma: changes in early mortality and outcomes in older patients. Leukemia. 2013;28(5):1122-1128.

5. Schaapveld M, Visser O, Siesling S, Schaar CG, Zweegman S, Vellenga E. Improved survival among younger but not among older patients with Multiple Myeloma in the Netherlands, a population-based study since 1989. Eur J Cancer. 2010;46(1):160-169. 
6. Fried LP, Ferrucci L, Darer J, Williamson JD, Anderson G. Untangling the concepts of disability, frailty, and comorbidity: implications for improved targeting and care. J Gerontol A Biol Sci Med Sci. 2004; 59(3):255-263.

7. Wedding U, Honecker F, Bokemeyer C, Pientka L, Höffken K. Tolerance to chemotherapy in elderly patients with cancer. Cancer Control. 2007;14(1):44-56. Available from: http://www.ncbi.nlm.nih.gov/ pubmed/17242670. Accessed February 4, 2016.

8. Hamaker ME, Jonker JM, de Rooij SE, Vos AG, Smorenburg CH, van Munster BC. Frailty screening methods for predicting outcome of a comprehensive geriatric assessment in elderly patients with cancer: a systematic review. Lancet Oncol. 2012;13(10):e437-e444.

9. Hutchins LF, Unger JM, Crowley JJ, Coltman CA, Albain KS. Underrepresentation of patients 65 years of age or older in cancer-treatment trials. N Engl J Med. 1999;341(27):2061-2067.

10. Kyle RA, Therneau TM, Rajkumar SV, et al. Prevalence of monoclonal gammopathy of undetermined significance. $N$ Engl $J$ Med. 2006;354(13): 1362-1369.

11. Tuchman SA, Shapiro GR, Ershler WB, et al. Multiple myeloma in the very old: an IASIA conference report. J Natl Cancer Inst. 2014; 106(5):1-9.

12. Palumbo A, Anderson K. Multiple Myeloma. N Engl J Med. 2011; 364:1046-1060.

13. Kyle RA, Gertz MA, Witzig TE, et al. Review of 1027 patients with newly diagnosed multiple myeloma. Mayo Clin Proc. 2003;78:21-33.

14. Howell DA, Smith AG, Jack A, et al. Time-to-diagnosis and symptoms of myeloma, lymphomas and leukaemias: a report from the Haematological Malignancy Research Network. BMC Hematol. 2013;13(1):9.

15. Lyratzopoulos G, Neal RD, Barbiere JM, Rubin GP, Abel GA. Variation in number of general practitioner consultations before hospital referral for cancer: findings from the 2010 National Cancer Patient Experience Survey in England. Lancet Oncol. 2012;13(4):353-365.

16. Greipp PR, Miguel JS, Dune BG, et al. International staging system for multiple myeloma. J Clin Oncol. 2005;23(15):3412-3420.

17. Bird JM, Owen RG, D'Sa S, et al. Guidelines for the diagnosis and management of multiple myeloma 2011. Br J Haematol. 2011;154(1): $32-75$.

18. Avet-Loiseau H, Hulin C, Campion L, et al. Chromosomal abnormalities are major prognostic factors in elderly patients with multiple myeloma: the intergroupe francophone du myélome experience. J Clin Oncol. 2013;31(22):2806-2809.

19. Riccardi A, Mora O, Tinelli C, et al. Long-term survival of stage I multiple myeloma given chemotherapy just after diagnosis or at progression of the disease: a multicentre randomized study. Cooperative group of study and treatment of multiple myeloma. $\mathrm{Br} J$ Cancer. 2000;82(7):1254-1260.

20. Kyle RA, Remstein ED, Therneau TM, et al. Clinical course and prognosis of smoldering (asymptomatic) multiple myeloma. $N$ Engl J Med. 2007;356(25):2582-2590.

21. Palumbo A, Bringhen S, Mateos M, et al. Geriatric assessment predicts survival and toxicities in elderly myeloma patients: an International Myeloma Working Group report. Blood. 2015;125(13): 2068-2075.

22. Antonio P, Valeria M, Sara B, et al. A randomized phase 3 trial of melphalan-lenalidomide-prednisone (MPR) or cyclophosphamideprednisone-lenalidomide (CPR) vs lenalidomide plus dexamethsone (Rd) in elderly newly diagnosed multiple myeloma patients. Presented at: ASH Annual Meeting Abstracts 536; December 9, 2013, Ernest N Morial Convention Center, New Orleans, LA.

23. Larocca A, Cavallo F, Magarotto V, et al. Reduced dose-intensity subcutaneous bortezomib plus prednisone (VP) or plus cyclophosfamide (VCP) or plus melphalan (VMP) for newly diagnosed multiple myeloma patients older than 75 years of age. Blood. 2013;122(21): 539-539.

24. Bringhen S, Petrucci MT, Larocca A, et al. Carfilzomib, cyclophosphamide, and dexamethasone in patients with newly diagnosed multiple myeloma: a multicenter, phase 2 study. Blood. 2014;124(1):63-69.
25. Kleber M, Ihorst G, Groß B, et al. Validation of the Freiburg comorbidity index in 466 multiple myeloma patients and combination with the international staging system are highly predictive for outcome. Clin Lymphoma Myeloma Leuk. 2013;13(5):541-551.

26. Bonanad S, De la Rubia J, Gironella M, et al. Development and psychometric validation of a brief comprehensive health status assessment scale in older patients with hematological malignancies: the GAH scale. J Geriatr Oncol. 2015;6(5):353-361.

27. Extermann M, Aapro M, Bernabei R, et al. Use of comprehensive geriatric assessment in older cancer patients: recommendations from the task force on CGA of the International Society of Geriatric Oncology (SIOG). Crit Rev Oncol Hematol. 2005;55(3):241-252.

28. Pallis AG, Ring A, Fortpied C, et al. Eortc workshop on clinical trial methodology in older individuals with a diagnosis of solid tumors. Ann Oncol. 2011;22(8):1922-1926.

29. Mill WB, Griffith R. The role of radiation therapy in the management of plasma cell tumors. Cancer. 1980;45(4):647-652.

30. Morgan GJ, Davies FE, Gregory WM, et al. First-line treatment with zoledronic acid as compared with clodronic acid in multiple myeloma (MRC Myeloma IX): a randomised controlled trial. Lancet. 2010;376(9757):1989-1999.

31. Knudsen LM, Hippe E, Hjorth M, Holmberg E, Westin J. Renal function in newly diagnosed multiple myeloma - a demographic study of 1,353 patients. The Nordic Myeloma study group. Eur J Haematol. 1994;53(4):207-212.

32. Srkalovic G, Cameron MG, Rybicki L, Deitcher SR, KattkeMarchant K, Hussein MA. Monoclonal gammopathy of undetermined significance and multiple myeloma are associated with an increased incidence of venothromboembolic disease. Cancer. 2004;101(3): 558-566.

33. Palumbo A, Rajkumar SV, Dimopoulos MA, et al. Prevention of thalidomide- and lenalidomide-associated thrombosis in myeloma. Leukemia. 2008;22(2):414-423.

34. Larocca A, Cavallo F, Bringhen S, et al. Aspirin or enoxaparin thromboprophylaxis for patients with newly diagnosed multiple myeloma treated with lenalidomide. Blood. 2012;119(4):933-939.

35. Augustson BM. Early mortality after diagnosis of multiple myeloma: analysis of patients entered onto the United Kingdom medical research council trials between 1980 and 2002 - medical research council adult leukaemia working party. J Clin Oncol. 2005;23(36): 9219-9226.

36. Mark TM, Boyer A, Rossi AC, et al. Clapd (Clarithromycin, Pomalidomide, Dexamethasone) therapy in relapsed or refractory multiple myeloma. Blood. 2013;122(21):1955.

37. Benboubker L, Dimopoulos MA, Dispenzieri A, et al. Lenalidomide and dexamethasone in transplant-ineligible patients with myeloma. N Engl J Med. 2014;371(10):906-917.

38. San Miguel JF, Schlag R, Khuageva NK, et al. Bortezomib plus melphalan and prednisone for initial treatment of multiple myeloma. $N$ Engl J Med. 2008;28;359(9):906-917.

39. Palumbo A, Bringhen S, Larocca A, et al. Bortezomib-melphalanprednisone-thalidomide followed by maintenance with bortezomibthalidomide compared with bortezomib-melphalan-prednisone for initial treatment of multiple myeloma: updated follow-up and improved survival. J Clin Oncol. 2014;32(7):634-640.

40. Palumbo A, Hajek R, Delforge M, et al. Continuous lenalidomide treatment for newly diagnosed multiple myeloma. $N$ Engl J Med. 2012;366(19):1759-1769.

41. Morgan GJ, Davies FE, Gregory WM, et al. Cyclophosphamide, thalidomide, and dexamethasone (CTD) as initial therapy for patients with multiple myeloma unsuitable for autologous transplantation. Blood. 2011;118(5):1231-1238.

42. Fayers PM, Palumbo A, Hulin C, et al. Thalidomide for previously untreated elderly patients with multiple myeloma: meta-analysis of 1685 individual patient data from 6 randomized clinical trials. Blood. 2011;118(5):1239-1247. 
43. Miguel JF, Schlag R, Khuageva NK, et al. Persistent overall survival benefit and no increased risk of second malignancies with bortezomib-melphalan-prednisone versus melphalan-prednisone in patients with previously untreated multiple myeloma. J Clin Oncol. 2013;31(4):448-455.

44. Moreau P, Pylypenko H, Grosicki S, et al. Subcutaneous versus intravenous administration of bortezomib in patients with relapsed multiple myeloma: a randomised, phase 3, non-inferiority study. Lancet Oncol. 2011;12(5):431-440.

45. Niesvizky R, Flinn IW, Rifkin R, et al. Community-based phase IIIB trial of three UPFRONT bortezomib-based myeloma regimens. J Clin Oncol. 2015;33(33):3921-3929.

46. Rajkumar SV, Jacobus S, Callander NS, et al. Lenalidomide plus highdose dexamethasone versus lenalidomide plus low-dose dexamethasone as initial therapy for newly diagnosed multiple myeloma: an open-label randomised controlled trial. Lancet Oncol. 2010;11(1):29-37.

47. Avet-Loiseau H, Hulin C, Benboubker L, et al. Impact of cytogenetics on outcomes of transplant-ineligible patients with newly diagnosed multiple myeloma treated with continuous lenalidomide plus low-dose dexamethasone in the first (MM-020) trial. Blood. 2015; 126(23):730.

48. Durie B, Hoering A, Rajkumar SV, et al. Bortezomib, lenalidomide and dexamethasone vs lenalidomide and dexamethasone in patients (Pts) with previously untreated multiple myeloma without an intent for immediate autologous stem cell transplant (ASCT): results of the randomized phase III trial SWOGS0777. Blood. 2015; 126(23):25.

49. Magarotto V, Bringhen S, Musto P, et al. Doublet vs triplet lenalidomidecontaining regimens in newly diagnosed myeloma patients, younger or older than 75 years: subgroup analysis of a phase III study. Blood. 2014;124(21):2110.

50. Kastritis E, Palumbo A, Dimopoulos MA. Treatment of relapsed/ refractory multiple myeloma. Semin Hematol. 2009;46(2): 143-157.

51. Weber DM, Chen C, Niesvizky R, et al. Lenalidomide plus dexamethasone for relapsed multiple myeloma in North America. N Engl J Med. 2007;357:2133-2142.

52. Jagannath S, Barlogie B, Berenson JR, et al. Updated survival analyses after prolonged follow-up of the phase 2, multicenter CREST study of bortezomib in relapsed or refractory multiple myeloma. Br J Haematol. 2008;143(4):537-540
53. Arnulf B, Pylypenko H, Grosicki S, et al. Updated survival analysis of a randomized phase III study of subcutaneous versus intravenous bortezomib in patients with relapsed multiple myeloma. Haematologica. 2012;97(12):1925-1928.

54. Castelli R, Pantaleo G, Gallipoli P, et al. Salvage therapy with bortezomib and dexamethasone in elderly patients with relapsed/refractory multiple myeloma. Anticancer Drugs. 2015;26(10):1078-1082.

55. Stewart AK, Rajkumar SV, Dimopoulos MA, et al. Carfilzomib, lenalidomide, and dexamethasone for relapsed multiple myeloma. $N \mathrm{Engl}$ J Med. 2015;372(2):142-152.

56. Moreau P, Masszi T, Grzasko N, et al. Ixazomib, an investigational oral proteasome inhibitor (PI), in combination with lenalidomide and dexamethasone (IRd), significantly extends progression-free survival (PFS) for patients (Pts) with relapsed and/or refractory multiple myeloma (RRMM): the Ph. Blood. 2015;126(23):727.

57. Lokhorst HM, Plesner T, Laubach JP, et al. Targeting CD38 with daratumumab monotherapy in multiple myeloma. N Engl J Med. 2015; 373(13):1207-1219

58. Lonial S, Dimopoulos M, Palumbo A, et al. Elotuzumab therapy for relapsed or refractory multiple myeloma. $N$ Engl J Med. 2015;373(7): 621-631.

59. End of life care for adults [webpage on the Internet] National Institute for Health and Clinical Excellence (NICE) (2011). Available at: https:// www.nice.org.uk/guidance/service-delivery--organisation-and-staffing/ end-of-life-care. Accessed February 25, 2016.

60. San Miguel JF, Schlag R, Khuageva NK, et al. Persistent overall survival benefit and no increased risk of second malignancies with bortezomib-melphalan-prednisone versus melphalan-prednisone in patients with previously untreated multiple myeloma. J Clin Oncol. 2013;31(4):448-455.

61. Mateos MV, Oriol A, Martínez-López J, et al. Bortezomib, melphalan, and prednisone versus bortezomib, thalidomide, and prednisone as induction therapy followed by maintenance treatment with bortezomib and thalidomide versus bortezomib and prednisone in elderly patients with untreated multiple myelom. Lancet Oncol. 2010;11(10):934-941.

62. Rajkumar SV, Dimopoulos MA, Palumbo A et al. IMWG updated criteria for the diagnosis of multiple myeloma. The Lancet Oncology. 2014;15(12):e538-e548.
Clinical Interventions in Aging

\section{Publish your work in this journal}

Clinical Interventions in Aging is an international, peer-reviewed journal focusing on evidence-based reports on the value or lack thereof of treatments intended to prevent or delay the onset of maladaptive correlates of aging in human beings. This journal is indexed on PubMed Central, MedLine,

\section{Dovepress}

CAS, Scopus and the Elsevier Bibliographic databases. The manuscript management system is completely online and includes a very quick and fair peer-review system, which is all easy to use. Visit http://www.dovepress. com/testimonials.php to read real quotes from published authors. 